\title{
Magnetohydrodynamic equations for toroidal plasmas
}

\section{Asif}

Department of Physics, COMSATS Institute of Information Technology, Lahore, Pakistan; dr.muhammad.asif@gmail.com

Received 23 November 2009; revised 4 December 2009; accepted 30 December 2009.

\begin{abstract}
A set of reduced MHD equations is derived using the standard energy balance equation. By applying assumption of internal energy, i.e. $R^{2} u=$ const, a set of reduced magnetohydrodynamic equations are obtained for large aspect ratio, high $\beta$ tokamaks. These equations include all terms of the same or der as the toroidal effect and only involve three variables, namely the flux, stream function and internal energy.
\end{abstract}

Keywords: Magnetohydrodynamics; Aspect Ratio; Viscosity

\section{INTRODUCTION}

Magnetohydrodynamic (MHD) instabilities [1], such as tearing modes, play an important role in plasma behaviour of tokamaks. They may influence both the particle and the energy confinement and are considered to be one of the main reasons for disruptions. Hence they have been studied by many authors [2-4]. The method of reduced magnetohydrodynamic based on a large-aspect ratio expansion has provided a powerful method for linear and nonlinear numerical computations.

In previous studies [2-4], the density of plasma is assumed to be constant. This assumption is valid in cylindrical geometry because the divergence of velocity is of the order $\varepsilon^{3}$ with $\varepsilon$ being the ratio of the minor radius to the length of cylinder. Thus we can neglect the divergence of velocity in the continuity equation. By applying assumption of density, i.e. $R^{2} \rho=$ const, Ren et al. [5] has derived the reduced MHD Equations in toroidal geometry for large aspect ratio, low $\beta$ tokamaks. Since the divergence of velocity is of the order $\varepsilon^{2}$ in toroidal geometry the same order as the toroidal effect, and cannot be neglected. Hence the assumption of constant density does not agree satisfactorily with the continuity equation in toroidal geometry. The present paper extends this work to high $\beta$ tokamaks.
In this paper, by using a new assumption about internal energy that is, $R^{2} u=$ const a set of reduced MHD Equations is derived. Where $\mathrm{R}$ is major radius and $u$ is an internal energy. The reduced MHD Equations involve three variables: the flux, stream function and internal energy. The Equations can be used to calculate the nonlinear evolution of tearing modes for toroidal plasmas.

\section{REDUCED MHD EQUATIONS}

The basic MHD Equations are of the form

$$
\begin{aligned}
& \frac{3}{2} \frac{d P}{d t}+\frac{5}{2} P \nabla \cdot v=0 \\
& \rho \frac{\partial v}{\partial t}=-\nabla P+J \times B \\
& J=\nabla \times B \\
& \frac{\partial B}{\partial t}=-\nabla \times E \\
& E+v \times B=\eta J
\end{aligned}
$$

In MHD, if $u=\frac{P}{\gamma-1}$, denote internal energy than standard energy Balance Equation has the form

$$
\frac{d u}{d t}+\gamma u \nabla \cdot v=0
$$

For the sake of simplicity, we adopt the quasi-cylindrical coordinate $(r, \theta, \varphi)$ with $\varphi$ being the toroidal angle. By assuming the inverse aspect ratio $\varepsilon$ to be a small quantity, the ordering of the high $\beta$ tokamaks [4].

$$
\begin{gathered}
O(1)=B_{\varphi}, \frac{\partial}{\partial r}, \frac{1}{r} \frac{\partial}{\partial \varphi} \\
O(\varepsilon)=B_{\perp}, J_{\varphi}, v_{\perp}, \frac{\partial}{\partial t}, \frac{1}{R}, u \\
O\left(\varepsilon^{2}\right)=\tilde{B}_{\varphi}, J_{\perp}, v_{\varphi}
\end{gathered}
$$


where $B_{\varphi}=\frac{B_{0} R_{0}}{R}$ is the externally applied magnetic field with $R_{0}$ being the coordinate of the geometric center of plasma and $B_{0}=\left.B_{\varphi}\right|_{R=R_{0}} . \quad \tilde{B}_{\varphi}$ is the toroidal magnetic field produced by plasma current. $J_{\varphi}$ is the toroidal current and $v_{\varphi}$ the toroidal velocity. The subscript $\perp$ denotes perpendicular to $\nabla \varphi$. The $u$ is the internal energy assumed to be of the order $\varepsilon$, for high $\beta$ tokamaks. For simplicity we use $B_{\varphi}$ to express $R B_{\varphi}, \quad \tilde{B}_{\varphi}$ to $R \tilde{B}_{\varphi}, \quad J_{\varphi}$ to $R J_{\varphi}, \quad v_{\varphi}$ to $R v_{\varphi}$. Thus $B_{\varphi}$ is of the order $\varepsilon^{-1}, J_{\varphi}$ is of zeroth order while $\tilde{B}_{\varphi}$ and $v_{\varphi}$ are of the order $\varepsilon$. Furthermore we assume $\eta$ to be of the order $\varepsilon^{2}$, for high $\beta$ tokamaks [4].

Introducing the vector potential $A$ for magnetic field $B$, that is,

$$
A=A_{r} e_{r}+A_{\vartheta} e_{\vartheta}+A \nabla \varphi
$$

Then $B$ can be expressed as

$$
=\nabla A \times \nabla \varphi-\frac{1}{R}\left(\frac{\partial A_{\vartheta}}{\partial \varphi} e_{r}-\frac{\partial A_{r}}{\partial \varphi} e_{\vartheta}\right)+\left(B_{\varphi}-\frac{R}{r} \frac{\partial A_{r}}{\partial_{\vartheta}}+R \frac{\partial A_{\vartheta}}{\partial r}\right) \nabla \varphi
$$

From Eq.(8) one can see that $A$ is of zeroth order and $A_{r}$ and $A_{\vartheta}$ are of the order $\varepsilon^{2}$. Compared with equilibrium magnetic filed expressed as $B=$ $\nabla \psi \times \nabla \varphi+\left(B_{\varphi}+\tilde{B}_{\varphi}\right) \nabla \varphi$, where $A$ is the flux function. Later it will be shown that the effect of $A_{r}$ and $A_{\vartheta}$ is of the order of $\varepsilon^{2}$ and can be neglected so that within our approximation $B$ can be determined by $A$ only. Substituting Eqs.(8) and (5) into Eq.(4) we obtain

$$
\nabla \times \frac{\partial A}{\partial t}=\nabla \times(v \times B)-\eta \nabla \times J, \frac{\partial A}{\partial t}=(v \times B)-\eta J+\nabla \phi
$$

where $\phi$ is the gauge potential. Eq.(9) can be deduced as

$$
-\frac{B_{\varphi}}{R^{2}} v_{\perp}+\nabla \phi \times \nabla \varphi=O\left(\varepsilon^{4}\right)
$$

Hence we can introduce stream function $U$. for $v$

$$
U=\frac{\phi}{B_{0}},
$$

where $U$ is the order $\varepsilon$ and $v$ can be expressed as

$$
v=\frac{R^{2}}{R_{0}} \nabla U \times \nabla \varphi+v_{\varphi} \nabla \varphi+O\left(\varepsilon^{3}\right)
$$

where the relation $B_{\varphi}=B_{0} R_{0}$ is used. The divergence of $v$ is obtained as

$$
\nabla . v=\frac{1}{R_{0}} \nabla R^{2} \times \nabla U . \nabla \varphi+O\left(\varepsilon^{3}\right) .
$$

This Eq.(12) shows that the divergence of $v$ is of the order $\varepsilon^{2}$. Taking the $\phi$ component of Eq.(9) and substituting Eqs.(10) and (12) into Eq.(9), we obtain the flux evolution equation

$$
\frac{\partial A}{\partial t}=\frac{R^{2}}{R_{0}} B . \nabla U+O\left(\varepsilon^{2}\right) .
$$

Taking the curl of $B$, we get the expression of current

$$
J_{\varphi}=R^{2} \nabla \varphi \cdot(\nabla \times B)=-R^{2} \nabla \cdot\left(R^{-2} \nabla A\right)+O\left(\varepsilon^{2}\right)
$$

where the $\varphi$-derivatives in operator are of the higher order and can be dropped. From the internal energy evolution Eq.(6) by substituting Eq.(11), we obtain

$$
\frac{d}{d t}\left(R^{2} u\right)=O(\varepsilon)
$$

The term on the right-hand side of Eq.(15) can be neglected because the term on the left-hand side is of zeroth order. This indicates that if we assume the internal energy to be constant, Eq.(15) cannot be satisfied. We assume

$$
R^{2} u=R_{0}^{2} u_{0}=\text { const }
$$

This assumption satisfies Eq.(15) and includes the toroidal effect.

Taking the curl of momentum Eq.(2) after multiplying by $R^{2}$ we can eliminate $J_{\perp}$ and $B_{\varphi}$. Then we get

$$
\rho_{0} \frac{d W}{d t}=-\frac{R^{2}}{R_{0}} B . \nabla J_{\varphi}+(\gamma-1) \frac{R^{2}}{R_{0}} \nabla R^{2} \times \nabla u . \nabla \varphi+O\left(\varepsilon^{2}\right)(17)
$$

where

$$
\begin{gathered}
W=R^{2} \nabla^{2} U, \\
\frac{d}{d t} \approx \frac{\partial}{\partial t}+\frac{R^{2}}{R_{0}} \nabla U \times \nabla \varphi . \nabla .
\end{gathered}
$$

The $\varphi$-derivatives in the Laplacian are also of the higher order and can be neglected. Using the Assumption of Eq.(16) we can directly obtain the parallel viscosity equation. Then a set of reduced MHD equations can be written as

$$
\frac{\partial A}{\partial t}=\frac{R^{2}}{R_{0}} B . \nabla U
$$

$$
\begin{gathered}
\rho_{0} \frac{d W}{d t}=-\frac{R^{2}}{R_{0}} B \cdot \nabla J_{\varphi}+(\gamma-1) \frac{R^{2}}{R_{0}} \nabla R^{2} \times \\
\frac{d}{d t}\left(R^{2} u\right)=0 \\
J_{\varphi}=-R^{2} \nabla \cdot\left(R^{-2} \nabla A\right) \\
W=R^{2} \nabla^{2} U,
\end{gathered}
$$


The Assumption 16 that the product of the square of the major radius and the internal energy is a constant, is quite stringent as taking this to be a constant, the first driving term in the Grad-Shafranov equation becomes just proportional to the flux derivative of the logarithm of the major radius, which shows a rather weak dependence. Therefore, this assumption seems to drastically narrow down the range of equilibrium configurations to which it is applicable. On the other hand, it has been observed [6] that a high density region appears near the inside limiter, which means that the density profile at the inside and outside of plasma along a flux surface is asymmetric. The pressure is calculated as the product of experimental temperature and density. Since the internal energy is related to the pressure as $u=\frac{P}{\gamma-1}$, we can say that the pressure distribution is nonuniform poloidally and the pressure is higher at the inside of plasma than at the outside. The result is, however, consistent with our expectation. On the other hand, density and pressure profile widths are clearly correlated [7].

\section{CONCLUSIONS}

In summary, we derived the reduced MHD Equations (18-22) by using the Assumption 16 about the internal energy in a large aspect ratio limit. These equations in clude all terms of the same order as the toroidal effect and only involve three variables, namely the flux, stream function and internal energy. These equations can be used to investigate the time evolution of tearing mode for the high $\beta$, large aspect ratio limit for tokamak Plasmas.

\section{REFERENCES}

[1] Wesson, J.A. (1978) Hydromagnetic stability of tokamaks. Nuclear Fusion, 18(1), 87-132.

[2] Fitzpatrick, R., Hastie, R.J., Martin, T.J. and Roach, C.M. (1993) Stability of coupled tearing modes in tokamaks. Nuclear Fusion, 33(10), 1533-1556.

[3] Strauss, H.R. (1983) Finite-aspect-ratio MHD equations for tokamaks. Nuclear Fusion, 23(5), 649-655.

[4] Strauss, H.R. (1977) Dynamics of high $\beta$ tokamaks. Physics Fluids, 20(8), 1354-1360.

[5] Ren, S.M. and Yu, G.Y. (2001) Reduced equations in toroidal geometry. Chinese Physics Letters, 18(4), 556-558.

[6] Frigione, D. et al. (1996) High density operation on frascati tokamak upgrade. Nuclear Fusion, 36(11), 14891499.

[7] Minardi, E. et al. (2001) Stationary magnetic entropy in ohmic tokamak plasmas: Experimental evidence from the TCV device. Nuclear Fusion, 41(1), 113-130. 